\title{
Introducing an Adolescent Cognitive Maturity Index and Tracking Vulnerability in Emerging Adulthood
}

\author{
Shady El Damaty ${ }^{1,2 *}$, Valerie L. Darcey ${ }^{1,2}$, Goldie A. McQuaid ${ }^{2}$, Maria Stoianova ${ }^{2}$, \\ Veronica Mucciarone $^{2}$, Yewon Chun ${ }^{2}$, Marissa L. Laws ${ }^{1}$, Victor Campano ${ }^{2}$, Kinney Van \\ Hecke $^{2}$, Mary Ryan ${ }^{2}$, Emma J. Rose, Diana H. Fishbein ${ }^{3,4}$ \& John W. VanMeter ${ }^{1}$ \\ ${ }^{1}$ Interdisciplinary Program in Neuroscience, Georgetown University, Washington, District of Columbia, USA ${ }^{2}$ Center for \\ Functional \& Molecular Imaging, Georgetown University Medical Center, Department of Neurology, Washington, District of \\ Columbia, USA ${ }^{3}$ Program for Translational Research on Adversity and Neurodevelopment (P-TRAN), Bennett-Pierce \\ Prevention Research Center, The Pennsylvania State University, University Park, PA $16802{ }^{4}$ Translational Neuro-Prevention \\ Research, Frank Porter Graham Child Development Institute, University of Northern Carolina, Chapel Hill, NC \\ Acknowledgments Thank you to Melissa Avalos, Amanda Patterson, Maysa Jawdat, Rachel Schroeder, Jaclyn Leiser, Paige \\ Trojanowski, Tomas Clarke, Kelly Martin, Macy Curell, Brittany Eltman, Dana Estefan, and Ileana Pacheco-Colon for \\ running MRI sessions, collecting behavioral data, and coordinating study procedures.
}

\begin{abstract}
Variation in maturational trajectories of adolescent neurocognitive development has been hypothesized to contribute to adverse outcomes in the transition to adulthood, such as experiencing violence. However, there remains a lack of consensus on the normative trajectory of cognitive maturation in adolescence. To address this problem, we derive a Cognitive Maturity Index (CMI), to estimate the difference between chronological and cognitive age predicted with latent factor estimates of inhibitory control, risky decision-making and emotional processing measured with standard neuropsychological instruments. Age prediction with latent factor estimates of cognitive skills approximated age within \pm 10 months $(r=0.71)$. Males in advanced puberty displayed lower cognitive maturity relative to peers of the same age; manifesting as weaker inhibitory control, greater risk taking, desensitization to negative affect, and poor recognition of positive affect. Elevated risk for future violent outcomes was effected by delayed CMI and fully mediated by drive for achieving rewards, illustrating adolescent maturation as a risk traversal process into young adulthood.
\end{abstract}

Keywords: adolescence, cognitive development, age prediction, maturity, dual systems model, maturational imbalance mode

\section{Introduction}

Cognitive Development in Emerging Adulthood The transition from adolescence to emerging adulthood is marked by the maturation of core cognitive control skills required for adaptive social decision-making in the execution of goal-directed behavior. These skills include active maintenance of goal-related representations in working memory, task switching, inhibitory control of reflexive behavior, weighing of risks versus rewards, and processing emotional context (Luna et al., 2015). The development of these cognitive skills during adolescence co-occurs with increased sensitivity to reinforcement signals in the form of positive or negative outcomes during social and general task learning (Jones et al., 2014; Rosenbaum et al., 2020). Social signals, such as facial expressions, have been demonstrated to attract automatic attention, modulate hedonia, and serve as reinforcers of socially desired behaviors (Teufel et al., 2009; Speer et al., 2007). Strong emotional context during social reinforcement learning captures attention and increases the speed and accuracy of learning new associations (Vernetti et al., 2017; Roper et al., 2014). Along this vein, several complementary models of adolescent brain development describe adolescence as a period of mismatch between the efficiency of cognitive control and affective salience of reinforcers (Casey et al., 2008; Luna and Wright, 2016). These models are corroborated by behavioral and neuroimaging evidence confirming that cognitive 
skills, and the brain regions that support them, develop together during adolescence. However, the exact timing of neurocognitive skill maturation and the interaction between cognitive control, risk taking, and emotion processing is not yet fully elucidated (Shulman et al., 2016; Duell et al., 2016). The Dual Systems Model claims inhibitory control increases monotonically whereas reward processing is an inverted u-shaped curve that briefly peaks in mid-adolescence and returns to baseline by adulthood. In contrast, the Maturational Imbalance Model proposes inhibitory control and reward processing develop well into adulthood and that greater inhibitory control may temper reward sensitivity by suppressing reflexive responses to hedonic stimuli. Neurophysiological and behavioral evidence supports both models. However, a key distinction to help settle the controversy can be made by tracking the maturation of cognitive skills from adolescence into emerging adulthood (Steinberg, 2010; Somerville, 2016).

Maturity and Risk for Violence Epidemiological data clearly illustrate that growing up, in-and-of-itself, is the greatest risk factor for a non-health related adverse outcome throughout the lifespan (Turner et al., 2006). In 2008, approximately $60 \%$ of youth under the age of 17 had experienced assault, sexual abuse, childhood maltreatment or either dating, domestic or community violence (Finkelhor, 2009), statistically portraying the transition to young adulthood as a formidable period of vulnerability during development (Berzin, 2010). Several lines of research on biomarkers of age-related change have demonstrated maturation is a complex transformational event influenced by biological and environmental mechanisms, which can vary significantly across individuals. Social adversity, in particular, has been shown to accelerate sexual maturation (Rickard et al., 2014), drive puberty-related epigenetic moderators of depressive symptoms (Sumner et al., 2019), engender developmental decreases in gray matter volume and corresponding increases in white matter fractional anisotropy (Gur et al., 2019; Gogtay et al., 2004), as well as alter development of functional brain network topology (Tooley et al., 2020) and cognitive skills (McLaughlin et al., 2019; Belsky, 2019). The maturational timing of risk-reward processing and inhibitory control skills is hypothesized to uniquely interact with the social pressures associated with achieving independence in emerging adulthood. Elevated risk taking, tenacious reward pursuit, weak inhibitory control and fluid social norm perceptions have been hypothesized to enhance the risk of violence experience in young adulthood (Morgan et al., 2014; Hasking, 2007). Disruptions to a normative maturational trajectory such as an over-saturation of negative reinforcers may overwhelm cognitive control resources over time, consequently, leading to poor adjustment, anti-social attitudes, and the increased likelihood of experiences of violence (Raine and Yang, 2006; Inzlicht et al., 2015). Criminology researchers have called attention to the importance of tracking development to better understand paths leading to violence experience, but the connection between models of adolescent cognitive maturation and the risk for adverse outcomes has yet to be demonstrated (Laub and Sampson, 1993).

Estimating Cognitive Age Although existing models of adolescent cognitive development have been helpful for understanding the transition to adulthood, a key missing aspect is a data-driven operational definition of neurocognitive age or maturity. A more precise definition has the potential to help distinguish sources of variation in development and help translate science for utilitarian social applications by identifying critical developmental windows during which particular interventions may exert the greatest benefits (Somerville, 2016). Early biological models of age based on DNA methylation, transcription and telomere length (Baker III and Sprott, 1988; Jylhävä et al., 2017), brain structure (Khundrakpam et al., 2015; Aycheh et al., 2018; Madan and Kensinger, 2018), and brain network oxygen metabolism (Dosenbach et al., 2010; Qin et al., 2015) have exhibited significant success in predicting age and identifying individual developmental trajectories bench-marked against the average growth curve in the sampled population. However, cognitive age prediction using theory-driven indicator variables obtained from behavioral experiments has yet to be implemented.

Limitations of Model-Free Approach The absence of a complete theoretical model of biological mechanisms underlying age-related changes has been coincident with a proliferation of analytic techniques using large numbers of features to extrapolate sample-specific effects (Sagers et al., 2020; Crimmins et al., 2008). Common methods for age prediction typically use big-data-driven approaches, involving the collection of large amounts of data per individual, such as genome-wide RNA transcription, or MRI measurements analyzed using hundreds of thousands of pair-wise correlations between voxels at discrete time intervals. These methods pose an issue in that there are significantly more descriptive features per individual than there 
are samples in the dataset (i.e., 'the curse of dimensionality'; $p>>n$, Taylor (2019)) - a problem that commonly leads to overfitting with standard linear regression. This issue is overcome through the application of data reduction and variable selection techniques, such as principal component analysis or regularized regression, that penalize or eliminate redundant features (Lee and Yoon, 2017). Model-free techniques have been successful for predicting age within an acceptable error range (Cole and Franke, 2017); however, these models do not always lend themselves to interpretation because variable selection and data reduction can be biased by their cost function, leading to overfitting (Babyak, 2004), or confounded by collinear indicators of age, such as motion in fMRI experiments (Satterthwaite et al., 2013).

Conceptual Prior for Modeling Maturity A potential remedy for the curse of dimensionality in age prediction is to build models with data derived from experiments designed to isolate and measure features of age-related change. Currently, there are no applications utilizing behavioral tasks originally developed for testing dual-systems-type models with the goal of estimating cognitive age in typically developing adolescents. Here, we describe a method for computing a cognitive maturity index (CMI) using reaction time, task performance, and other derived metrics from the Continuous Performance (CPT), Wheel of Fortune (WOF), Emotional Face Recognition (EFR), and Temporal Delay Discounting (TD) tasks collected in the Adolescent Development Study (Fishbein et al., 2016). The social utility of CMI is demonstrated by using estimated cognitive maturity as a causal predictor of risk for violent outcomes in emerging adulthood. First, confirmatory Factor Analysis (CFA) is used to estimate directly unobservable latent cognitive factors predicted to change with age during adolescence; such as inhibitory control, risk/reward processing, and emotion recognition. The interaction between factors is tested in a structural equation model (SEM) and latent factor estimates of cognitive skills used as predictors in a regularized regression model to predict age. The CMI is defined as the difference between observed and predicted cognitive age for each participant and explored as an indicator of developmental risk for violent outcomes in young adulthood. A high CMI indicates accelerated cognitive maturity relative to the sample mean, whereas a low CMI indicates a relatively lagging developmental trajectory. In our sample, low CMI predicted elevated risk for future violent outcomes in puberty advanced males, which was mediated by behavioral drive for achieving rewards. This work demonstrates that predicting cognitive age using latent constructs is a promising technique that can be scaled with larger neurocognitive datasets to generate more accurate population estimates for adolescent neurocognitive maturity and further illuminate the interaction between social context and trajectories of neurocognitive development.

\section{Methods}

\section{Participants}

A cross-section of early adolescent youth were recruited to participate in the Adolescent Development Study (ADS), a prospective, longitudinal investigation of the neurodevelopmental factors underlying early substance use initiation and the consequences on brain development (Fishbein et al., 2016). Youth $(N=141)$ from the Washington, D.C. Metro area were enrolled in 2011 (Table 1). As of March 2020, participants have completed up to four sequential waves of data collection separated by approximately 18 months. Eligibility at wave 1 included the following criteria: 1) ages 11-13 years, 2) right-handedness, 3) no history of neuropsychiatric disorders or recent head injuries, 4) no self-reported consumption of one or more units of alcohol or other substances and 5) not of direct Asian descent. A total of six participants were excluded following the baseline visit due to: substance use at baseline $(N=2)$, autism diagnosis $(N=1)$, and high scores on ambidexterity measured with the Edinburgh Handedness Test $(N=3)$, (Veale, 2014). Despite attrition, the distribution of sex and race remained approximately the same throughout the study $(+/-0.5 \%)$. Participants were compensated and reimbursed for travel, when applicable. All youth and caregivers gave their informed assent and consent prior to data collection and study procedures were reviewed and approved by the Georgetown University Institutional Review Board. 


\section{Interview Procedure and Collected Metrics}

Pre-screened, eligible participants were invited for an on-site visit at Georgetown University Medical Center to complete a series of questionnaires and interviews designed to measure neurocognitive developmental traits and capture social and family life. The accompanying primary caregiver was interviewed in a separate room and asked to complete a questionnaire regarding economic status, education, and the difficulty of acquiring basic needs such as food, healthcare, and housing (Bornstein et al., 2003). Socioeconomic Status (SES) was estimated from these responses by converting the family's household income to z-scores, averaging both guardian/parents' years of education, converting the average to a z-score, and lastly averaging the income and education z-scores for the final SES measure (Manuck et al., 2010).

Anthropometrics Adolescents had their height and weight measured and completed a pubertal development scale (Carskadon and Acebo, 1993) to measure body-mass index (BMI) and physical changes with age at each wave. Pubertal development scores were derived from from reported changes in height, body hair, complexion, vocal pitch, breast size, and menarche. Respondents responded with: "has not yet begun," "has barely begun," "is definitely underway," or "is complete" for each puberty related physical feature queried by the instrument.

Instruments Risk for future adverse outcomes (i.e., substance abuse, mood disorders, delinquent behavior) and future violence proneness was measured with the revised Drug Use Screening Inventory (DUSI-R; 153 items) in waves one through three (Tarter et al., 1994). The DUSI-R is composed of ten subscales: substance abuse, behavioral problems, psychiatric disturbances, medical problems, family dysfunction, work problems, school maladjustment, social skills deficiency, peer relationship problems, and maladaptive leisure and recreation activities. A set of ten items designed to detect spurious responses (i.e., choosing no for all questions to speed through the visit) are nested within the instrument, composing a lie scale for identifying outlier response patterns. Participants with a cumulative lie score $>6$ are excluded as their responses are considered to be inaccurate and/or invalid (Dalla-Déa et al., 2003). Here, the DUSI-R was used to compute a metric for Violence Proneness (DUSI-VP) — an estimation of the risk of experiencing violence in early adulthood, either as a victim or perpetrator (Kirisci et al., 2009). The Violence Proneness metric reflects the average of the school (seven items) and peer relations problems (six items) subscales. All participants were also assessed for verbal and performance IQ using the Kaufman Brief Intelligence Test (KBIT), (Kaufman, 2004). Finally, adolescents completed The Behavioral Inhibition System/Behavioral Activation System (BIS/BAS) Scale to provide a measure of appetitive and avoidant behavioral tendencies (Carver and White, 1994). The BAS is divided into three subscales measuring funseeking (BAS-FS; four items, ex. "I will often do things for no other reason than that they might be fun."), independent drive (BAS-D; four items, ex. "I go out of my way to get things I want") and reward responsivity (BAS-RR; five items, ex. "When I get something I want, I feel excited and energized") used to measure self-reported idiosyncratic differences in temperament and personality underlying reinforcement sensitivity (Corr, 2004).

\section{Neurocognitive Tasks}

Participants completed the Continuous Performance and Wheel of Fortune tasks while undergoing functional MRI (Siemens Tim Trio 3T Scanner) during waves one through three. Stimuli were projected onto a screen and reflected into the participant's field of view using a mirror. Participants responded using fiber optical button boxes. The Emotional Face Recognition and Temporal Discounting tasks were completed outside of the scanner on a laptop in a private behavioral testing room in the first three waves. The Facial Emotion Recognition Task was administered with E-Prime 1.2. All other neurocognitive tasks were built and presented with the E-prime Stimulus Presentation Software Version 2.0 (Schneider et al., 2002).

Continuous Performance Task (CPT) The continuous performance task was used to measure impulsivity and inhibitory control of reflexive actions (Horn et al., 2003). Participants viewed five blocks of 30 letters presented one-at-a-time for a 200ms duration (150 total trials). Each block was separated by a "cool-down" period in which a gray fixation cross was presented for $1300 \mathrm{~ms}$. Participants were instructed to press the right-hand button box as quickly as possible for all letters except "Q". The lure 
"Q" appeared 27 times in the task. The sequence of letters was the same across all participants. Signal Detection Theory metrics were utilized for analyzing CPT behavior Stanislaw and Todorov (1999). 'Hits' were defined as correct button presses to target letters and 'Misses' as failure to respond to a target letter. 'False-Alarms' were defined as an incorrect response to the the lure "Q", whereas 'Correct Rejections' were defined as correct inhibition of response. Discriminative sensitivity to lures was measured by the $d^{\prime}$ variable, $d^{\prime}=\phi^{-1}$ (Hits $)-\phi^{-1}$ (FalseAlarms), and response-bias calculated by utilizing the natural log-transformed beta estimate, $\beta=0.5 *\left[\phi^{-1}(\text { FalseAlarms })^{2}-\phi^{-1}(\text { Hits })^{2}\right]$; where $\phi^{-1}$ is the inverse probability distribution function (Forbes et al., 2011).

Wheel of Fortune Task (WOF) A modified version of the Wheel of Fortune (WOF) task was administered to test propensity of risk-taking and gambling strategies (Ernst et al., 2004). The task was divided into three runs of 30 trials. In each trial, a "wheel" appeared on the screen that portrayed the probabilities of winning different amounts of virtual money. Participants were instructed to select between large monetary gains with a low probability (high-risk) or small monetary gains with high probability (low-risk) and indicated their choice using button boxes placed in their left and right hands (corresponding to choice of the left and right sides of the wheel, respectively). Winning probability varied pseudo-randomly between a $10: 90 \%$ split (occurring 32 to 42 times of a total 90 trials) and a $30: 70 \%$ split (occurring 48 to 58 times). The quantities of money assigned to the left and right side of the wheel varied between a $\$ 1$ - $\$ 9$ for the low risk choice or a $\$ 2$ - $\$ 18$ split for the high risk choice, when the wheel was split $10: 90$. The quantities similarly varied between $\$ 3$ - $\$ 7$ or a $\$ 9$ - $\$ 21$ split when the wheel was divided $30: 70$. These values and proportional assignments assured that the expected value (EV) appeared equal for a winning selection independent of risk (e.g., $30 \%$ chance of winning $\$ 7=\$ 2.10 \mathrm{EV}$ vs. $70 \%$ chance of winning $\$ 3=\$ 2.10 \mathrm{EV}$ ). Spatial position of the rewards varied evenly, with the larger reward appearing on each side $50 \%$ of the time. The wheel was visible until the participant made their selection, or for a maximum of $3000 \mathrm{~ms}$, followed by a $3000 \mathrm{~ms}$ delay after which feedback was presented indicating whether the participant had won or lost the selected dollar amount, along with their cumulative winnings up to and including that trial. Participants automatically lost the higher dollar amount if no decision was made before $3000 \mathrm{~ms}$ had elapsed. Each run began with a $6000 \mathrm{~ms}$ fixation, and the inter-trial interval was varied based on a Poisson distribution between 2500 to $10000 \mathrm{~ms}$. The total quantity of money won or lost would be reset to $\$ 0$ at the beginning of each run. Participants were encouraged to improve upon the amount they won in the next run. Participants were encouraged to respond as if their gains and losses were real, however no real money or physical reward was given. Task performance was analyzed using the probability of high vs. low risk decisions, the reaction time to make those decisions and the cumulative winnings at each run. Anticipatory responses were defined as trials $<200 \mathrm{~ms}$ reaction time and discarded from analysis as outliers.

Emotional Face Recognition Task (EFR) Participants viewed 70 photographs (grayscale images, $284 \mathrm{x}$ 351 pixels, resolution $=96 \mathrm{dpi}$ ) from the NimStim dataset, which includes images of twenty-nine professional actors aged 21-30 years (12 female, 17 male, 14 European-American, 10 African-American, 3 Asian-American 2 Latino-American) instructed to pose for expressions of seven emotions: happiness, surprise, sadness, anger, disgust, fear and neutral (Tottenham et al., 2009). To prepare for the task, participants were presented showcards with labels of each of seven emotions and asked to describe each of the emotions followed by a practice trial for each emotion. For each trial, a photograph appeared for a maximum duration of 5000ms along with seven labels for each of the emotions. Participants were instructed to click on the emotion with a mouse to advance to the next trial. The image disappeared after $5000 \mathrm{~ms}$ and labels remained until a response was made. Trials were separated by an inter-trial interval of $3000 \mathrm{~ms}$, during which time participants viewed a white screen. No actor was shown with the same emotion more than once. The accuracy and reaction time for disgust, anger, sadness and fear were averaged together to compose estimates of performance for recognizing negative emotion. Positive emotion recognition performance was derived from reaction time and accuracy for happy faces only.

Temporal Delay Discounting Task (TD) Individual preference between small, immediate vs. large, delayed rewards was tested by offering participants a forced choice between two options: "Would you rather have $\$ X$ now or $\$ X+Y$ in $Z$ days?" The delay of rewards, $Z$, was varied from zero, one, two, ten days, 1 
month, half a year and one year. The immediately available amount, $X$, was determined using a random adjustment procedure that updated the current choice based on previous choices. Immediate reward amounts varied from $\$ 0.50$ to $\$ 10$ while the delayed reward value $X+Y$ was fixed at $\$ 10$. The propensity for discounting the objective value due to delayed receipt was computed as the area under the curve (AUC) using the trapezoidal method: $A U C=\sum_{t=0}^{t=k}\left(d_{t+1}-d_{t}\right) *\left(v_{t}+v_{t+1} / 2\right)$, where $t$ is the current normalized delay point, $k$ the maximum delay and $v$ the indifference value at that point (Borges et al., 2016; Olson et al., 2007; Myerson et al., 2001). Indifference points reflecting the subjective value of the immediately available reward at a given delay were normalized to the maximum award value of $\$ 10$ and plotted across annualized time delays. As described above, trapezoids formed by normalized subjective values at each normalized delay contributed to AUC in the range from $0-1$. A large AUC indicates participants are more likely to prefer large but delayed rewards whereas a lower AUC indicates preference for immediate gratification. In order to elicit behavior reflective of the participant's actual preferences, participants were informed that they would receive either a $\$ 5$ or $\$ 10$ reward based randomly on their choices in the task prior to the task.

\section{Statistical Analysis}

Data were converted from double-entered paper records, Qualtrics survey exports, and E-prime task outputs then consolidated into a single long-format data frame containing an observation for each participant at each wave. Next, descriptive statistics and assessments of multivariate normality were performed with the Arsenal (3.5.0), MVN (5.8, Korkmaz et al. (2014)) and DescTools (0.99.36, Signorell (2020)) packages in R version Orange Blossom (1.2.5033, R Core Team (2019)). Participants with high DUSI-R Lie scores ( $\mathrm{N}=23$; Lie> 6) were excluded from analysis (Dalla-Déa et al., 2003). Demographics for the excluded participants did not significantly differ from the retained group. Scored neurocognitive and sociodemographic measures were correlated with age to identify and confirm expected bivarate relationships with development, including that of age-related changes in neurocognitive skills as revealed by EFR, CPT, WoF, TD task performance. Confirmatory Factor Analysis (CFA) was implemented with the Lavaan R package (0.6-6) to estimate latent factors for inhibitory control, risk/reward processing, and emotional face recognition (Rosseel, 2012). Latent factor analysis is a statistical method for estimating an underlying mechanism that can only be indirectly measured through indicators derived from experimental constructs (Finch and French, 2015). Latent factor estimates are obtained by averaging the unique contribution of each indicator variable after controlling for the shared variance across indicators to satisfy the local independence principle (Sobel, 1997). The resulting estimate is considered as a weighted combination of the residual covariance between indicator variables used to approximate the inferred latent factor, where the correlation between two indicator variables is permitted when they are both caused by the underlying latent variable (Cooper et al., 2019). This approach serves to reduce the number of variables required to model a hypothesized cognitive phenomenon and helps minimize the contribution of residual error on tests of statistical inference; both useful and necessary requirements for building predictive regression models that avoid over-fitting with collinear predictors. Maximum likelihood estimation with full information maximum likelihood (FIML) was used to adjust CFA parameter estimates for missing data (Cham et al., 2017). The latent factors were standardized to allow for free estimation of factor loadings and post hoc testing (Hu and Bentler, 1998). The model fit of the implied structural relationships was assessed with $\chi^{2}$ Goodness of Fit referenced against a null model with 0 factor loadings, the Root Mean Square Error of Approximation (RMSEA), the Comparative Fit Index (CFI) and the Tucker-Lewis Index (TLI; Kenny et al. (2015); Hu and Bentler (1999); Wu et al. (2009)). Path and structural models were visualized with the semPlot $\mathrm{R}$ package (1.1.2). The standardized latent factor estimates of inhibitory control, risk taking and facial emotion recognition were used to predict age with regularized linear regression models implemented in the glmnet $\mathrm{R}$ package (4.0-2) using leave-one-out cross-validation for estimation of model hyperparameters and a 50\% random split across participants between training and test datasets (Friedman et al., 2010, 2009). Cross-validated model performance was estimated by computing the $R^{2}$ from the mean cross-validated error divided by the variance of observed age in the test sample across the regularization rate $(\lambda)$ and penalty factor $(\alpha ; 0=$ Ridge Regression, $0.5=$ Elastic Net, $1=$ Least Absolute Shrinkage and Selection Operator) hyper-parameter estimates. The minimum $\lambda$ at the highest $R^{2}$ was used to calculate regression coefficients in the training sample. The cognitive maturity index was estimated as the difference in the observed and predicted age for each participant. 


\section{Results}

\section{Latent Factor Analysis of Cognitive Metrics}

Inhibitory Control The inhibitory control latent factor (ICLF) was characterized by consistently careful responses and greater target discrimination in the CPT, along with overall behavioral caution measured by the BIS. The ICLF was estimated using the CPT response time standard deviation, log transformed response bias, $\ln (\beta)$, target discrimination, $d^{\prime}$, and the BIS part of the BIS/BAS. Response time standard deviation was selected for the model a priori because mean reaction time did not significantly vary trialby-trial across participants except at the beginning of a block (Figure S1). Additionally, participants were instructed to press the response button as fast as possible and variations in responses were typically only observed circa false alarm trials during which more cautious participants would modify their pace to avoid errors. Task performance improved from wave one to three independent of task learning effects (Figure $\mathrm{S} 2, \mathrm{~S} 3)$. The fitted relations were significant compared to a null model with 0 factor loadings between all measures, residual covariances, and the latent variable estimate (Table 2; CLI $=1.0 ; T L I=1.018$; $R M S E A<0.01, p=0.91)$. Greater discriminative ability between false alarms and targets was significantly inversely correlated to response bias $(-0.46 \pm 0.14 ; Z=3.36 ; p=0.001)$ and was associated with greater variation in response time to targets $(0.30 \pm 0.09 ; Z=3.36 ; p=0.001)$, suggesting participants modified their behavior and responded with more consistent timing as they learned the task (Figure 1). This was corroborated by a strong linear relationship between $d^{\prime}$ and the average response time to targets (Figure S4; $0.57 \pm 0.05 ; Z=12.11, p<0.001)$. Response time variance to targets interacted inversely with false alarm response time to predict $d^{\prime}(-0.08 \pm 0.02 ; Z=-3.35, p<0.001)$ indicating that rapid response to early false alarms led to greater caution later in the task, resulting in overall improvement in target/lure discrimination and CPT performance. Response time variation was uncorrelated between target and false alarm trials $(0.07 \pm 0.09 ; Z=0.70, p=0.48)$ demonstrating that each provided a statistically independent contribution to ICLF. The significant contribution of the BIS to estimation of ICLF revealed that inhibitory control in the CPT was also indicative of generic internalizing behavior and disposition towards caution. The ICLF did not vary by sex, or BMI, but was found to significantly increase with SES $(0.267 \pm 0.053$; $Z=4.99, p<0.001)$ and pubertal development $(0.14 \pm 0.06 ; Z=2.22, p=0.03)$.

Risk Taking The risk/reward evaluation latent factor (RRLF) was found to drive impulsive risk taking and a preference for immediate rewards. The RRLF was estimated using probability of high risk decisions, cumulative winnings and response time in the WOF task, and area under the curve of TD performance. Overall, RRLF was characterized by greater risky decisions, moderate response time for high risk options, longer response time for low risk decisions, significantly poorer cumulative winnings and stronger propensity for immediate rewards in the TD task (Table 3). The model fit to the data significantly improved by allowing free estimation of covariance between the probability of making a high risk decision and response time, and between low and high risk response times. The fitted relations with these free parameters revealed a significant fit of the covariance structure compared to a null model with 0 factor loadings between all measures, residual covariances, and the RRLF estimate $(C L I=1.0 ; T L I=1.0 ; R M S E A=0.005, p=$ 0.846). The estimate of the covariance between response time and the probability of making a high high risk decision revealed that risky decisions occurred more quickly than carefully evaluated low risk options $(-0.121 \pm 0.031 ; Z=-3.849, p<0.001)$. Overall, the RRLF was related to greater risk taking in the WOF task, which resulted in poor cumulative winnings due to high probability of loss and consequently running a negative balance. RRLF did not significantly covary with sex, PDS, BMI, or SES.

Emotional Face Recognition Performance in the EFR task was used to derive latent factors of positive and negative emotional face recognition (EPLF/ENLF). Latent factors were estimated using the mean and standard deviation of response time and accuracy to recognize angry, fearful, sad, or disgusted (negative) and happy (positive) facial expressions. The model fit was improved significantly by permitting free covariation between mean reaction time to negative and positive faces, negative and positive emotion recognition accuracy, and between standard deviation and mean response time for both negative and positive emotions. The proposed model structure explained a significant proportion of the covariance between task metrics and provided an excellent fit compared to a null model (Table 4; CLI $=0.978 ; T L I=0.942 ; R M S E A=0.073$, 
$p=0.115)$. Perceptual processing of positive emotional faces often resulted in correct emotion recognition, but was associated with generally longer and more variant time to recognition, reflecting difficulty in distinguishing between happy and other facial expressions. Perceptual processing of negative emotional faces often resulted in correct recognition with quick and consistently low variation in recognition time. The structural equation model revealed perceptual processing performance for negative emotions was inversely related to positive emotion processing (standardized estimate $-3.44 \pm 1.85 ; Z=-1.86, p=0.063$ ). This result suggests participants generally tuned to facial expressions of negative affect were more likely to exhibit rapid and inaccurate recognition of positive affect. ENLF, but not EPLF, was found to increase with SES $(0.16 \pm 0.07$; $Z=2.11, p=0.036)$ and BMI $(0.04 \pm 0.02 ; Z=2.65, p=0.008)$, suggesting youth from prosperous, more educated, households are more sensitive to recognizing facial expressions of negative affect but do not possess comparable performance on positive affect recognition. No significant relationship between ENLF and sex or PDS was revealed. Greater physical maturation measured with PDS was related to lower EPLF scores $(-0.70 \pm 0.15 ; Z=-4.55, p<0.001)$, indicating that physically mature youth exhibit faster, less variable, recognition time and poorer accuracy for happy facial expressions. No relationship between EPLF and SES, BMI, or sex was found.

\section{Structural Equation Model of Cognitive Factors}

A structural equation model was constructed to explore the interaction between the identified latent factors and their indicator variables (Figure 2). Significant covariations were identified between the constituent cognitive latent factors compared to a null model $(C L I=0.95 ; T L I=0.93 ; R M S E A=0.047, p=0.64)$. ICLF was found to significantly reduce RRLF, suggesting greater inhibitory control can curtail a propensity for risk taking $(-0.22 \pm 0.10 ; Z=-2.16, p<0.031)$. Greater inhibitory control also predicted greater sensitivity to negative emotional faces $(0.55 \pm 0.26 ; Z=2.09, p=0.037)$ and effected faster recognition time for all emotions at the expense of recognizing happy facial expressions. As previously noted, elevated ENLF was inversely correlated with EPLF. No relationship was found between RRLF and negative or positive emotional processing.

\section{Cognitive Maturity Index}

Linear ordinary least squares regression was used to test ICLF, RRLF, EPLF, and ENLF as predictors of chronological age to explore the utility of building a predictive model of neurocognitive age. Inhibitory control $(0.72 \pm 0.01 ; Z=7.50, p<0.001)$ and negative affect perceptual processing $(0.35 \pm 0.14 ; Z=2.44$, $p=0.015)$ significantly increased in efficiency with age, whereas risky reward taking $(-0.22 \pm 0.10 ; Z=-2.35$, $p=0.019)$ and positive affect recognition $(-0.16 \pm 0.10 ; Z=-1.52, p=0.129)$ declined. Ridge regression $(\alpha=0, \lambda=0.083)$ with latent factors predicted age in a split-half test sample within a mean absolute error of \pm 10.11 months $\left(R^{2}=0.51\right.$; Figure 3$)$, significantly more accurately than by training on the original indicator variables used to generate the latent variable estimates $\left(R^{2}=0.16\right)$. The difference between predicted and observed age, defined as the cognitive maturity index (CMI), was computed for each participant. A lower CMI value indicated delayed maturity and a higher value indicated accelerated maturity of cognitive skills, relative to the average development of in-sample youth at a given age. Greater CMI correlated with lower BMI (Pearson's $R=-0.24, p<0.001)$, slower pubertal development $(R=-0.20, p<0.001$ ), lower BAS-D $(R=-0.16, p<0.001)$, greater performance IQ scores $(R=0.20, p<0.001)$, and lower DUSI-R problem scale scores on: substance use $(R=-0.20, p<0.001)$, health risk $(R=-0.16, p<0.001)$, and, lastly, risk for violence $(R=-0.28, p<0.001)$. No significant relationship was found between SES and CMI, or between any of the other BIS/BAS scales and CMI. Advanced pubertal development $(-0.67 \pm 0.089 ; Z=-7.47$, $p<0.001)$ interacted with sex $(-2.44 \pm 0.39 ; Z=-6.21, p<0.001)$ to predict greater CMI in more fully developed females compared to males (interaction effect estimate $0.82 \pm 0.16 ; Z=5.30, p<0.001$ ).

\section{Vulnerability for Future Violent Outcomes}

Path analysis was implemented to test the hypothesis that tenacity for reward pursuit, measured with the BAS "drive" subscale (BAS-D), mediated the relationship between CMI and developmental violence risk from ages 11-18 (Figure 4). A full mediation between CMI, BAS-D, and risk for future violent outcomes was 
identified (5). A higher CMI was associated with depressed BAS-D $(-0.40 \pm 0.14 ; Z=-3.73 ; p<0.001)$, which in turn interacted with elevated CMI to predict lower violence risk. A post-hoc ANOVA revealed that lower EPLF $(-0.020 \pm 0.01 ; Z=-2.62, p=0.009)$ and lower SES $(-0.36 \pm 0.15 ; Z=-2.50, p=0.01)$ independently predicted elevated risk for future violent outcomes $(F=3.107, p=0.002)$. No other latent cognitive factor predicted risk independently, however a trending interaction between cognitive latent factors was related to elevated $\operatorname{risk}(-0.014 \pm 0.001 ; Z=-1.947, p=0.052)$.

\section{Discussion}

The distinction between chronological and biological age has been explored in great detail but not yet extended to account for different trajectories of neurocognitive maturation during adolescence. The current study shows development of an improved method for identifying behavioral hallmarks of cognitive development that can be used to estimate population-relative maturity in adolescents and has significant implications for predicting adverse life outcomes in emerging adulthood. Theory-driven variable reduction was implemented with regularized regression for optimized age-prediction accuracy ( \pm 10 months) than has been previously possible with neuroimaging datasets ( \pm 1 year), which typically incorporate a massive number of features, leading to overfitting (Cole and Franke, 2017; Franke et al., 2012). Estimated maturity was found to be a significant effector of DUSI-R violence risk status in adolescence, an association that was mediated by the behavioral drive for desired goals and rewards in the transition to adulthood. Youth at elevated risk were likely to live in less wealthy and educated households and exhibit delayed maturity of cognitive skills such as lower inhibitory control, desensitization to negative emotion facial expressions, and greater risk taking. The observed outcomes underscore the importance of framing adolescent cognitive development within the greater social environmental context.

Adolescent Cognitive Development Models of adolescent neurocognitive development have reached a general consensus that risk taking and cognitive control change significantly during the maturational transition from childhood to adulthood (Steinberg, 2010). However, agreement on the dynamics and timing of this transition has remained elusive. The Dual Systems Model describes the approach to maturity as a linear age-related increase in cognitive control that catches up over time with the inverted u-shaped development of the reward system (Steinberg, 2005). Elevated risk-taking and reward sensitivity are considered to be transient events in the transition to adulthood before returning to a pre-adolescent baseline. In contrast, the Maturational Imbalance Model suggests both the reward and cognitive control systems develop exponentially over time, though at different rates, and reach a stable plateau that continues into adulthood (Casey et al., 2008). The early emergence of elevated risk/reward processing is considered to be tempered by later maturation of cognitive control in the Maturational Imbalance Model (Somerville and Casey, 2010). Further elaborations of these models have included an additional emotional processing component that peaks mid-adolescence and overwhelms cognitive control by enhancing the weighting of emotionally salient reward representations during decision making (Casey et al., 2019).

Latent Cognitive Factors In this study, distinctions between the Dual Systems and Maturational Imbalance models were explored by modeling cognitive skills, measured by a battery of neurocognitive tasks, as unobserved latent factors. Behavioral data collected during performance of the CPT, WOF, and EFR tasks were modeled with confirmatory factor analysis to reveal latent factors illustrating inhibitory control, risk/reward processing, and positive and negative emotion perceptual processing. Inhibitory control was found to reflect greater proactive control of motor reflexes in a rapid response task along with elevated cautionary decision-making, as queried by the BIS scale of the BIS/BAS instrument. Inhibitory control did not covary by sex but was found to correlate positively with SES, suggesting that the presence/absence of social opportunity may temper/promote impulsive decision-making, respectively. Risk/reward processing was an indicator of greater, more impulsive decisions for immediate rewards - especially more-so when perceived losses were greater and high risk was concurrent with high rewards. Risk/reward processing was expected to be inversely correlated with SES, but no significant relationship was found in the tested sample. Structural 
equation modeling of the interaction between cognitive latent factors showed elevated inhibitory control, resulted in lower risky decision making, demonstrating that a greater degree of inhibitory control was reflected as a lower propensity for risk taking; providing support for the Maturational Imbalance model. Inhibitory control was also correlated with greater negative emotion perceptual processing skill, corroborating that adolescent cognitive development is concurrent with a greater sensitivity to negative social reinforcers (such as negative emotional context, or in this study faces of disgust, anger or fear; Jones et al. (2014); Rosenbaum et al. (2020)). Negative and positive emotional face processing were found to be inversely correlated - meaning that high sensitivity (fast and accurate responses) to negative emotions is reflected with fast and less accurate recognition of positive emotions. As with elevated inhibitory control, sensitivity to negative emotions was found to be significantly greater in higher SES youth. Overall, our approach demonstrates that latent factors underlying cognitive skills development in adolescence can be estimated with standard well-validated cognitive tasks and instruments. Structural equation modeling revealed significant interactions between latent cognitive factors supporting the Maturational Imbalance model, where inhibitory control is expected to increase monotonically with age while tempering risk taking into adulthood. The results highlight the importance of including cognitive processing of emotion in models of adolescent brain development. A normative development of inhibitory control was revealed to be concordant with emotion recognition ability and sensitivity bias to perceptions of negative emotional faces. Socioeconomic status was a significant covariate of cognitive skills development and calls attention to the importance of attending to social environmental context in models of adolescent neurocognitive development.

Decomposition of Cognitive Maturity Latent factor analysis provided best estimates of cognitive control skills for building a predictive model of adolescent neurocognitive maturity compared to the use of standard behavioral metrics (i.e., response time, performance) for predicting age. Improved estimation is credited to the minimization of measurement error through enforcement of local independence during latent factor estimation. The interactions between ICLF, RRLF, ENLF and EPLF were preserved as a function of age-related change. The CMI is a normalized estimate computed at each wave relative to the mean expected age in the sample and thus is an indicator of maturity only in the context of the sampled population. A greater CMI indicated more advanced development, whereas a lower CMI suggested lagging development of cognitive skills relative to the sample mean. Pubertal development (PDS) and sex were significant effectors of cognitive maturation. Females exhibited earlier/faster pubertal development and accelerated cognitive maturation (i.e., greater inhibitory control, less risk taking, emotion sensitization). Males with higher scale of physical development were more likely to score higher on the BAS-D, a measure of tenacity and desire for achieving goals and receiving rewards, and show delayed CMI compared to their peers.

Cognitive Maturity and Vulnerability for Violent Outcomes Criminologists have identified violence risk status in adolescence to be tightly related to age-related change. However there is no clear connection between the timing of cognitive maturity and vulnerability for adverse outcomes. We explored the relationship between cognitive skills development and vulnerability by fitting a causal structural equation model between estimated cognitive maturity, drive for rewards, and adolescent risk for violence experience in adulthood. The relationship between CMI and risk for violent outcomes was found to be fully mediated by the BAS-D, suggesting that elevated desire for receiving rewards may increase the violence risk for male adolescents exhibiting lower inhibitory control, elevated risk taking and desensitization to facial expressions of emotion. Of the latent cognitive factors, EPLF was the strongest predictor of DUSI-R risk for a violent outcome by adulthood; where poor recognition of positive emotions correlated with a higher level of risk. All together, these results suggest the relative trajectory of cognitive skills development may interact with social status and sex to influence the risk of adverse outcomes in adulthood and demonstrate the significant implications of maturity index for tracking adolescent vulnerability.

Conclusions A lack of consensus regarding the dynamics of cognitive skills development and their effect on adverse outcomes in emerging adulthood is compounded by the difficulty of defining when adolescence ends and adulthood begins in regards to neurocognitive development. In this work, we apply open-source statistical methods to provide a simple first step for approximating individual-specific neurocognitive age with linear latent factor modeling of inhibitory control, risk taking, and emotional face perceptual processing skills. 
Further, we demonstrate the translational utility of CMI as a predictor of youth violence. This approach embraces the perspective that maturity is best modeled as a relative factor and occurs along a continuum well into the early 20s. In other words, estimations of the neurocognitive maturity of an individual should take into account the contextual elements of their environment, peer-related influences, and psychosocial developmental history. Such a definition has exceptionally vital social implications, most notably having been used as supporting evidence in the 2005 Roper v.s. Simmons Supreme Court proceedings weighing against the death penalty for minors. Here we demonstrate sample-relative estimates of maturity can be derived from behavioral performance on neurocognitive instruments and used to model risk factors for adverse outcomes in adolescence. Future work can apply this method with larger datasets, such as the ABCD project (Casey et al., 2018; Volkow et al., 2018), to better approximate a population estimate of adolescent neurocognitve maturity. Fusion with neuroimaging data may also serve to augment estimates by including biological mechanisms, to better delineate interactions between environmental and biological contributions to cognitive skills development.

\section{Conflict of Interest Statement}

The authors declare that the research was conducted in the absence of any commercial or financial relationships that could be construed as a potential conflict of interest.

\section{Author Contributions}

Shady El Damaty wrote the manuscript, analyzed the data, devised the analytic method for CMI, and collected data for wave four under mentorship from Diana H. Fishbein, Emma R. Jane and John W. VanMeter. Valerie L. Darcey, Goldie A. McQuaid and Veronica Mucciarone collected waves one through three data and made significant contributions to the manuscript. Valerie L. Darcey was vital for scoring and interpreting the temporal delay discounting task and Goldie A. McQuaid for documenting the Facial Emotion Recognition task. Maria Stoianova contributed to the manuscript, was responsible for importing paper records to RedCap, and managed data automation efforts for this project. Yewon Chun, Kinney Van Hecke and Mary Ryan contributed to the manuscript, data collection and coding wave four data for analysis. Marissa L. Laws and Victor Campano contributed to the manuscript, completed the coding of, and also contributed to the analysis of, the Wheel of Fortune task.

\section{Funding}

This research was supported by the following grants NIH/NIAAA 1R01AA019983-01 and 3R01AA01998302S1, NIH/NCATS 1KL2RR031974-01, NIH/NICHD 2P30HD040677-11 and OJP 2016-90111-DC-IJ

\section{Acknowledgments}

Thank you to Melissa Avalos, Amanda Patterson, Maysa Jawdat, Rachel Schroeder, Jaclyn Leiser, Paige Trojanowski, Tomas Clarke, Kelly Martin, Macy Curell, Brittany Eltman, Dana Estefan, and Ileana PachecoColon for running MRI sessions, collecting behavioral data, and coordinating study procedures.

\section{Supplemental Data \& Data Availability}

The datasets and supplement for this study can be found at https://osf.io/cdyxh/. 


\section{References}

Aycheh, H. M., Seong, J.-K., Shin, J.-H., Na, D. L., Kang, B., Seo, S. W., and Sohn, K.-A. (2018). Biological brain age prediction using cortical thickness data: A large scale cohort study. Frontiers in aging neuroscience, 10:252.

Babyak, M. A. (2004). What you see may not be what you get: a brief, nontechnical introduction to overfitting in regression-type models. Psychosomatic medicine, 66(3):411-421.

Baker III, G. T. and Sprott, R. L. (1988). Biomarkers of aging. Experimental gerontology, 23(4-5):223-239.

Belsky, J. (2019). Early-life adversity accelerates child and adolescent development. Current Directions in Psychological Science, 28(3):241-246.

Berzin, S. C. (2010). Vulnerability in the transition to adulthood: Defining risk based on youth profiles. Children and youth services review, 32(4):487-495.

Borges, A. M., Kuang, J., Milhorn, H., and Yi, R. (2016). An alternative approach to calculating areaunder-the-curve (auc) in delay discounting research. Journal of the Experimental Analysis of Behavior, 106(2):145-155.

Bornstein, M. H., Hahn, C.-S., Suwalsky, J. T., and Haynes, O. M. (2003). Socioeconomic status, parenting, and child development: The Hollingshead Four-Factor Index of Social Status and The Socioeconomic Index of Occupations. Lawrence Erlbaum Associates Publishers.

Carskadon, M. A. and Acebo, C. (1993). A self-administered rating scale for pubertal development. Journal of Adolescent Health, 14(3):190-195.

Carver, C. S. and White, T. L. (1994). Behavioral inhibition, behavioral activation, and affective responses to impending reward and punishment: the bis/bas scales. Journal of personality and social psychology, $67(2): 319$.

Casey, B., Cannonier, T., Conley, M. I., Cohen, A. O., Barch, D. M., Heitzeg, M. M., Soules, M. E., Teslovich, T., Dellarco, D. V., Garavan, H., et al. (2018). The adolescent brain cognitive development (abcd) study: imaging acquisition across 21 sites. Developmental cognitive neuroscience, 32:43-54.

Casey, B., Heller, A. S., Gee, D. G., and Cohen, A. O. (2019). Development of the emotional brain. Neuroscience letters, 693:29-34.

Casey, B. J., Getz, S., and Galvan, A. (2008). The adolescent brain. Developmental review, 28(1):62-77.

Cham, H., Reshetnyak, E., Rosenfeld, B., and Breitbart, W. (2017). Full information maximum likelihood estimation for latent variable interactions with incomplete indicators. Multivariate behavioral research, $52(1): 12-30$.

Cole, J. H. and Franke, K. (2017). Predicting age using neuroimaging: innovative brain ageing biomarkers. Trends in neurosciences, 40(12):681-690.

Cooper, S. R., Jackson, J. J., Barch, D. M., and Braver, T. S. (2019). Neuroimaging of individual differences: A latent variable modeling perspective. Neuroscience \& Biobehavioral Reviews, 98:29-46.

Corr, P. J. (2004). Reinforcement sensitivity theory and personality. Neuroscience 83 Biobehavioral Reviews, $28(3): 317-332$.

Crimmins, E., Vasunilashorn, S., Kim, J. K., and Alley, D. (2008). Biomarkers related to aging in human populations. Advances in clinical chemistry, 46:161-216.

Dalla-Déa, H. R. F., De Micheli, D., and Formigoni, M. L. O. S. (2003). Effects of identification and usefulness of the lie scale of the drug use screening inventory (dusi-r) in the assessment of adolescent drug use. Drug and alcohol dependence, $72(3): 215-223$. 
Dosenbach, N. U., Nardos, B., Cohen, A. L., Fair, D. A., Power, J. D., Church, J. A., Nelson, S. M., Wig, G. S., Vogel, A. C., Lessov-Schlaggar, C. N., et al. (2010). Prediction of individual brain maturity using fmri. Science, 329(5997):1358-1361.

Duell, N., Steinberg, L., Chein, J., Al-Hassan, S. M., Bacchini, D., Lei, C., Chaudhary, N., Di Giunta, L., Dodge, K. A., Fanti, K. A., et al. (2016). Interaction of reward seeking and self-regulation in the prediction of risk taking: A cross-national test of the dual systems model. Developmental psychology, 52(10):1593.

Ernst, M., Nelson, E. E., McClure, E. B., Monk, C. S., Munson, S., Eshel, N., Zarahn, E., Leibenluft, E., Zametkin, A., Towbin, K., et al. (2004). Choice selection and reward anticipation: an fmri study. Neuropsychologia, 42(12):1585-1597.

Finch, W. H. and French, B. F. (2015). Latent variable modeling with R. Routledge.

Finkelhor, D. (2009). Children's exposure to violence: A comprehensive national survey. Diane Publishing.

Fishbein, D., Rose, E., Darcey, V., Belcher, A., and VanMeter, J. (2016). Neurodevelopmental precursors and consequences of substance use during adolescence: Promises and pitfalls of longitudinal neuroimaging strategies. Frontiers in Human Neuroscience, 10:296.

Forbes, C., Evans, M., Hastings, N., and Peacock, B. (2011). Statistical distributions. John Wiley \& Sons.

Franke, K., Luders, E., May, A., Wilke, M., and Gaser, C. (2012). Brain maturation: predicting individual brainage in children and adolescents using structural mri. Neuroimage, 63(3):1305-1312.

Friedman, J., Hastie, T., and Tibshirani, R. (2009). glmnet: Lasso and elastic-net regularized generalized linear models. $R$ package version, $1(4)$.

Friedman, J., Hastie, T., and Tibshirani, R. (2010). Regularization paths for generalized linear models via coordinate descent. Journal of Statistical Software, 33(1):1-22.

Gogtay, N., Giedd, J. N., Lusk, L., Hayashi, K. M., Greenstein, D., Vaituzis, C. A., Nugent, T. F., Herman, D. H., Clasen, L. S., Toga, A. W., Rapoport, J. L., and Thompson, P. M. (2004). Dynamic mapping of human cortical development during childhood through early adulthood. P Natl Acad Sci Usa, 101:81748179.

Gur, R. E., Moore, T. M., Rosen, A. F., Barzilay, R., Roalf, D. R., Calkins, M. E., Ruparel, K., Scott, J. C., Almasy, L., Satterthwaite, T. D., et al. (2019). Burden of environmental adversity associated with psychopathology, maturation, and brain behavior parameters in youths. JAMA psychiatry, 76(9):966-975.

Hasking, P. A. (2007). Reinforcement sensitivity, coping, and delinquent behaviour in adolescents. Journal of Adolescence, 30(5):739-749.

Horn, N., Dolan, M., Elliott, R., Deakin, J. F., and Woodruff, P. (2003). Response inhibition and impulsivity: an fmri study. Neuropsychologia, 41(14):1959-1966.

Hu, L.-t. and Bentler, P. M. (1998). Fit indices in covariance structure modeling: Sensitivity to underparameterized model misspecification. Psychological methods, 3(4):424.

Hu, L.-t. and Bentler, P. M. (1999). Cutoff criteria for fit indexes in covariance structure analysis: Conventional criteria versus new alternatives. Structural equation modeling: a multidisciplinary journal, 6(1):1-55.

Inzlicht, M., Bartholow, B. D., and Hirsh, J. B. (2015). Emotional foundations of cognitive control. Trends in cognitive sciences, 19(3):126-132.

Jones, R. M., Somerville, L. H., Li, J., Ruberry, E. J., Powers, A., Mehta, N., Dyke, J., and Casey, B. (2014). Adolescent-specific patterns of behavior and neural activity during social reinforcement learning. Cognitive, Affective, \& Behavioral Neuroscience, 14(2):683-697.

Jylhävä, J., Pedersen, N. L., and Hägg, S. (2017). Biological age predictors. EBioMedicine, 21:29-36. 
Kaufman, A. S. (2004). Kaufman brief intelligence test-second edition (kbit-2). Circle Pines, MN: American Guidance Service.

Kenny, D. A., Kaniskan, B., and McCoach, D. B. (2015). The performance of rmsea in models with small degrees of freedom. Sociological Methods \&3 Research, 44(3):486-507.

Khundrakpam, B. S., Tohka, J., Evans, A. C., Group, B. D. C., et al. (2015). Prediction of brain maturity based on cortical thickness at different spatial resolutions. Neuroimage, 111:350-359.

Kirisci, L., Tarter, R., and Reynolds, M. (2009). The violence proneness scale of the dusi-r predicts adverse outcomes associated with substance abuse. American journal on addictions, 18(2):173-177.

Korkmaz, S., Goksuluk, D., and Zararsiz, G. (2014). Mvn: An r package for assessing multivariate normality. The $R$ Journal, 6(2):151-162.

Laub, J. H. and Sampson, R. J. (1993). Turning points in the life course: Why change matters to the study of crime. Criminology, 31(3):301-325.

Lee, C. H. and Yoon, H.-J. (2017). Medical big data: promise and challenges. Kidney research and clinical practice, $36(1): 3$.

Luna, B., Marek, S., Larsen, B., Tervo-Clemmens, B., and Chahal, R. (2015). An integrative model of the maturation of cognitive control. Annual review of neuroscience, 38:151-170.

Luna, B. and Wright, C. (2016). Adolescent brain development: Implications for the juvenile criminal justice system. APA handbook of psychology and juvenile justice.

Madan, C. R. and Kensinger, E. A. (2018). Predicting age from cortical structure across the lifespan. European Journal of Neuroscience, 47(5):399-416.

Manuck, S. B., Phillips, J., Gianaros, P. J., Flory, J. D., and Muldoon, M. F. (2010). Subjective socioeconomic status and presence of the metabolic syndrome in midlife community volunteers. Psychosomatic medicine, $72(1): 35$.

McLaughlin, K. A., Weissman, D., and Bitrán, D. (2019). Childhood adversity and neural development: a systematic review. Annual review of developmental psychology, 1:277-312.

Morgan, J. E., Bowen, K. L., Moore, S. C., and Van Goozen, S. H. (2014). The relationship between reward and punishment sensitivity and antisocial behavior in male adolescents. Personality and individual differences, 63:122-127.

Myerson, J., Green, L., and Warusawitharana, M. (2001). Area under the curve as a measure of discounting. Journal of the experimental analysis of behavior, 76(2):235-243.

Olson, E. A., Hooper, C. J., Collins, P., and Luciana, M. (2007). Adolescents' performance on delay and probability discounting tasks: contributions of age, intelligence, executive functioning, and self-reported externalizing behavior. Personality and individual differences, 43(7):1886-1897.

Qin, J., Chen, S.-G., Hu, D., Zeng, L.-L., Fan, Y.-M., Chen, X.-P., and Shen, H. (2015). Predicting individual brain maturity using dynamic functional connectivity. Frontiers in human neuroscience, 9:418.

R Core Team (2019). R: A Language and Environment for Statistical Computing. R Foundation for Statistical Computing, Vienna, Austria.

Raine, A. and Yang, Y. (2006). Neural foundations to moral reasoning and antisocial behavior. Social cognitive and affective neuroscience, 1(3):203-213.

Rickard, I. J., Frankenhuis, W. E., and Nettle, D. (2014). Why are childhood family factors associated with timing of maturation? a role for internal prediction. Perspectives on Psychological Science, 9(1):3-15. 
Roper, Z. J., Vecera, S. P., and Vaidya, J. G. (2014). Value-driven attentional capture in adolescence. Psychological science, 25(11):1987-1993.

Rosenbaum, G., Grassie, H., and Hartley, C. (2020). Valence biases in reinforcement learning shift across adolescence and modulate subsequent memory. PsyArXiv.

Rosseel, Y. (2012). lavaan: An R package for structural equation modeling. Journal of Statistical Software, $48(2): 1-36$.

Sagers, L., Melas-Kyriazi, L., Patel, C. J., and Manrai, A. K. (2020). Prediction of chronological and biological age from laboratory data. Aging (Albany NY), 12(9):7626.

Satterthwaite, T. D., Wolf, D. H., Ruparel, K., Erus, G., Elliott, M. A., Eickhoff, S. B., Gennatas, E. D., Jackson, C., Prabhakaran, K., Smith, A., et al. (2013). Heterogeneous impact of motion on fundamental patterns of developmental changes in functional connectivity during youth. Neuroimage, 83:45-57.

Schneider, W., Eschman, A., and Zuccolotto, A. (2002). E-Prime: User's guide. Psychology Software Incorporated.

Shulman, E. P., Smith, A. R., Silva, K., Icenogle, G., Duell, N., Chein, J., and Steinberg, L. (2016). The dual systems model: Review, reappraisal, and reaffirmation. Developmental cognitive neuroscience, 17:103-117.

Signorell, A. (2020). DescTools: Tools for Descriptive Statistics. R package version 0.99.37.

Sobel, M. E. (1997). Measurement, causation and local independence in latent variable models. In Latent variable modeling and applications to causality, pages 11-28. Springer.

Somerville, L. H. (2016). Searching for signatures of brain maturity: what are we searching for? Neuron, 92(6):1164-1167.

Somerville, L. H. and Casey, B. (2010). Developmental neurobiology of cognitive control and motivational systems. Current opinion in neurobiology, 20(2):236-241.

Speer, L. L., Cook, A. E., McMahon, W. M., and Clark, E. (2007). Face processing in children with autism: Effects of stimulus contents and type. Autism, 11(3):265-277.

Stanislaw, H. and Todorov, N. (1999). Calculation of signal detection theory measures. Behavior research methods, instruments, \&3 computers, 31(1):137-149.

Steinberg, L. (2005). Cognitive and affective development in adolescence. Trends in cognitive sciences, $9(2): 69-74$.

Steinberg, L. (2010). A dual systems model of adolescent risk-taking. Dev Psychobiol, 52:216-224.

Sumner, J. A., Colich, N. L., Uddin, M., Armstrong, D., and McLaughlin, K. A. (2019). Early experiences of threat, but not deprivation, are associated with accelerated biological aging in children and adolescents. Biological psychiatry, 85(3):268-278.

Tarter, R. E., Mezzich, A. C., Kirisci, L., and Kaczynksi, N. (1994). Reliability of the drug use screening inventory among adolescent alcoholics. Journal of Child \& Adolescent Substance Abuse, 3(1):25-36.

Taylor, C. (2019). Applications Of Dynamic Programming To Agricultural Decision Problems. CRC Press.

Teufel, C., Alexis, D. M., Todd, H., Lawrance-Owen, A. J., Clayton, N. S., and Davis, G. (2009). Social cognition modulates the sensory coding of observed gaze direction. Current Biology, 19(15):1274-1277.

Tooley, U. A., Mackey, A. P., Ciric, R., Ruparel, K., Moore, T. M., Gur, R. C., Gur, R. E., Satterthwaite, T. D., and Bassett, D. S. (2020). Associations between neighborhood ses and functional brain network development. Cerebral Cortex, 30(1):1-19. 
Tottenham, N., Tanaka, J. W., Leon, A. C., McCarry, T., Nurse, M., Hare, T. A., Marcus, D. J., Westerlund, A., Casey, B., and Nelson, C. (2009). The nimstim set of facial expressions: judgments from untrained research participants. Psychiatry research, 168(3):242-249.

Turner, H. A., Finkelhor, D., and Ormrod, R. (2006). The effect of lifetime victimization on the mental health of children and adolescents. Social science $\& 3$ medicine, 62(1):13-27.

Veale, J. F. (2014). Edinburgh handedness inventory-short form: a revised version based on confirmatory factor analysis. Laterality: Asymmetries of Body, Brain and Cognition, 19(2):164-177.

Vernetti, A., Smith, T. J., and Senju, A. (2017). Gaze-contingent reinforcement learning reveals incentive value of social signals in young children and adults. Proceedings of the Royal Society B: Biological Sciences, 284(1850):20162747.

Volkow, N. D., Koob, G. F., Croyle, R. T., Bianchi, D. W., Gordon, J. A., Koroshetz, W. J., Pérez-Stable, E. J., Riley, W. T., Bloch, M. H., Conway, K., et al. (2018). The conception of the abcd study: From substance use to a broad nih collaboration. Developmental cognitive neuroscience, 32:4-7.

Wu, W., West, S. G., and Taylor, A. B. (2009). Evaluating model fit for growth curve models: Integration of fit indices from sem and mlm frameworks. Psychological methods, 14(3):183. 


\begin{tabular}{|c|c|c|c|c|c|}
\hline & & Wave One & Wave Two & Wave Three & $\mathrm{P}(>\|\chi\|)$ \\
\hline \multirow[t]{3}{*}{ Sex } & & & & & 0.930 \\
\hline & Female & $65(53.60 \%)$ & $62(54.50 \%)$ & $60(54.0 \%)$ & \\
\hline & Male & $57(46.40 \%)$ & $54(45.5 \%)$ & $48(46.0 \%)$ & \\
\hline \multirow[t]{5}{*}{ Race } & & & & & 0.918 \\
\hline & White & $65(54.60 \%)$ & $62(55.40 \%)$ & $61(58.10 \%)$ & \\
\hline & Black & $37(31.10 \%)$ & $35(31.20 \%)$ & $29(27.60 \%)$ & \\
\hline & Hispanic & $8(6.70 \%)$ & $5(4.50 \%)$ & $4(3.80 \%)$ & \\
\hline & Other & $9(7.60 \%)$ & $10(8.90 \%)$ & $11(10.05 \%)$ & \\
\hline \multirow[t]{3}{*}{ Age } & & & & & $<0.001$ \\
\hline & Mean (SD) & $12.68(0.76)$ & $14.32(0.82)$ & $15.84(0.80)$ & \\
\hline & Range & $11.11-14.00$ & $12.41-16.12$ & $13.87-18.01$ & \\
\hline \multirow[t]{3}{*}{ BMI } & & & & & 0.004 \\
\hline & Mean (SD) & $21.01(4.73)$ & $22.00(5.04)$ & $23.14(5.15)$ & \\
\hline & Range & $14.40-45.46$ & $15.35-47.90$ & $15.59-46.17$ & \\
\hline \multirow[t]{3}{*}{ Composite IQ } & & & & & 0.380 \\
\hline & Mean (SD) & $110.66(14.25)$ & $108.03(15.26)$ & $109.02(13.13)$ & \\
\hline & Range & $75.00-139.00$ & $72.00-136.00$ & $84.00-138.00$ & \\
\hline \multirow[t]{3}{*}{ DUSI-VP } & & & & & $<0.001$ \\
\hline & Mean (SD) & $2.79(2.05)$ & $3.53(2.50)$ & $4.23(2.76)$ & \\
\hline & Range & $0.00-9.00$ & $0.00-10.00$ & $0.00-11.00$ & \\
\hline \multirow[t]{3}{*}{ BIS } & & & & & 0.395 \\
\hline & Mean (SD) & $20.12(3.30)$ & $20.34(3.67)$ & $20.78(3.92)$ & \\
\hline & Range & $12.00-28.00$ & $13.00-27.00$ & $10.00-28.00$ & \\
\hline \multirow[t]{3}{*}{ BAS-D } & & & & & 0.012 \\
\hline & Mean (SD) & $9.83(2.55)$ & $10.18(2.42)$ & $10.81(2.37)$ & \\
\hline & Range & $4.00-16.00$ & $5.00-16.00$ & $5.00-16.00$ & \\
\hline \multirow[t]{3}{*}{ BAS-FS } & & & & & 0.509 \\
\hline & Mean (SD) & $11.50(2.39)$ & $11.23(2.25)$ & $11.16(2.29)$ & \\
\hline & Range & $4.00-16.00$ & $5.00-16.00$ & $6.00-16.00$ & \\
\hline \multirow[t]{3}{*}{ BAS-RR } & & & & & 0.849 \\
\hline & Mean (SD) & $17.68(1.67)$ & $17.61(1.82)$ & $17.54(1.97)$ & \\
\hline & Range & $14.00-20.00$ & $12.00-20.00$ & $13.00-20.00$ & \\
\hline
\end{tabular}

Table 1: Demographic, risk, and neuropyschological indicators assessed across development. $\chi^{2}$ test revealed significant effect of Age, BMI, DUSI-VP and BAS-D across waves. A total of 23 unique participants were excluded from summary statistics at any wave due to high DUSI-LIE 


\begin{tabular}{lllll} 
Inhibitory Control Latent Factor & Estimate & Std. Error & Z-value & $\mathrm{P}(>\|z\|)$ \\
\hline CPT Target Discrimination (d') & 0.650 & 0.090 & 7.189 & $<0.001$ \\
CPT Response Bias $(\beta)$ & -0.503 & 0.124 & -4.059 & $<0.001$ \\
CPT Hit RT Standard Deviation & -0.913 & 0.115 & -7.910 & $<0.001$ \\
CPT False Alarm RT Standard Deviation & -0.371 & 0.085 & -4.367 & $<0.001$ \\
Behavioral Inhibition System (BIS) & 0.193 & 0.058 & 3.335 & 0.001
\end{tabular}

Table 2: Normalized estimates for latent factors estimated with structural equation modeling of inhibitory control using continuous performance task metrics and the behavioral inhibition system scale. $\mathrm{CFI}=1.00$, $\mathrm{TLI}=1.018, \mathrm{RMSEA}=0.001, \mathrm{p}=0.910$

\begin{tabular}{lllll} 
Reward/Risk Latent Factor & Estimate & Std. Error & Z-value & $\mathrm{P}(>\|z\|)$ \\
\hline WOF Percent High Risk Choices & 0.703 & 0.087 & 8.106 & $<0.001$ \\
WOF High Risk Mean Reaction Time & 0.158 & 0.060 & 2.648 & 0.008 \\
WOF Low Risk Mean Reaction Time & 0.433 & 0.073 & 5.971 & $<0.001$ \\
WOF Cumulative Winnings & -0.899 & 0.076 & -11.817 & $<0.001$ \\
Temporal Discounting & -0.127 & 0.064 & -1.974 & 0.048
\end{tabular}

Table 3: Normalized estimates for latent factors estimated with confirmatory factor analysis summarizing reward/risk taking in the Wheel of Fortune Gambling and Temporal Delay Discounting tasks. CFI=1.00, $\mathrm{TLI}=1.00, \mathrm{RMSEA}=0.005, \mathrm{p}=0.846$

\begin{tabular}{lllll} 
Negative Emotions Latent Factor & Estimate & Std. Error & Z-value & $\mathrm{P}(>\|z\|)$ \\
\hline EFR Accuracy & & & & \\
EFR Mean Reaction Time & 0.164 & 0.088 & 1.869 & 0.062 \\
EFR Standard Deviation of Reaction Time & -0.451 & 0.128 & -3.536 & $<0.001$ \\
& & 0.085 & -3.541 & $<0.001$ \\
Positive Emotions Latent Factor & & & & \\
\hline EFR Accuracy & & & & \\
EFR Mean Reaction Time & 0.173 & 0.088 & 2.245 & 0.092 \\
EFR Standard Deviation of Reaction Time & 0.310 & 0.132 & 2.702 & 0.048 \\
& & 0.093 & 2.818 & 0.042
\end{tabular}

Table 4: Normalized estimates for latent factors estimated with confirmatory factor analysis summarizing emotional face recognition task performance for positive and negative emotions. $\mathrm{CFI}=0.978$, TLI $=0.942$, RMSEA $=0.073, \mathrm{p}=0.115$ 


\begin{tabular}{llllll} 
Path Model Regression & & Estimate & Std.Err & Z-value & $\mathrm{P}(>\|z\|)$ \\
\hline DUSI-VP & & & & & \\
& Sex & -0.495 & 0.267 & -1.856 & 0.063 \\
(direct) & SES & -0.369 & 0.145 & -2.538 & 0.011 \\
(indirect) & CMI & -0.597 & 0.133 & -4.480 & $<0.001$ \\
& BAS-D & 0.122 & 0.056 & 2.164 & 0.030 \\
BAS-D & & & & & \\
(indirect) & CMI & -0.376 & 0.132 & -2.843 & 0.004 \\
CMI & & & & & \\
& & & & & \\
& Sex & -0.496 & 0.137 & -3.634 & $<0.001$ \\
Mediation Parameters & & -0.402 & 0.082 & -5.088 & $<0.001$ \\
& & & & & \\
& Total & -0.449 & 0.132 & -3.392 & 0.001 \\
& Direct & -0.376 & 0.132 & -2.843 & 0.004 \\
& Indirect & -0.073 & 0.034 & -2.142 & 0.032
\end{tabular}

Table 5: Mediation model of risk for violent outcomes in emerging adulthood. Regression path estimates appear for the best fit model. Estimation of total, direct and indirect paths from CMI through BAS-D to effect DUSI-VP show significant total mediation. $\mathrm{CFI}=1.00, \mathrm{TLI}=1.08, \mathrm{RMSEA}=<0.001, \mathrm{p}=0.912$ 

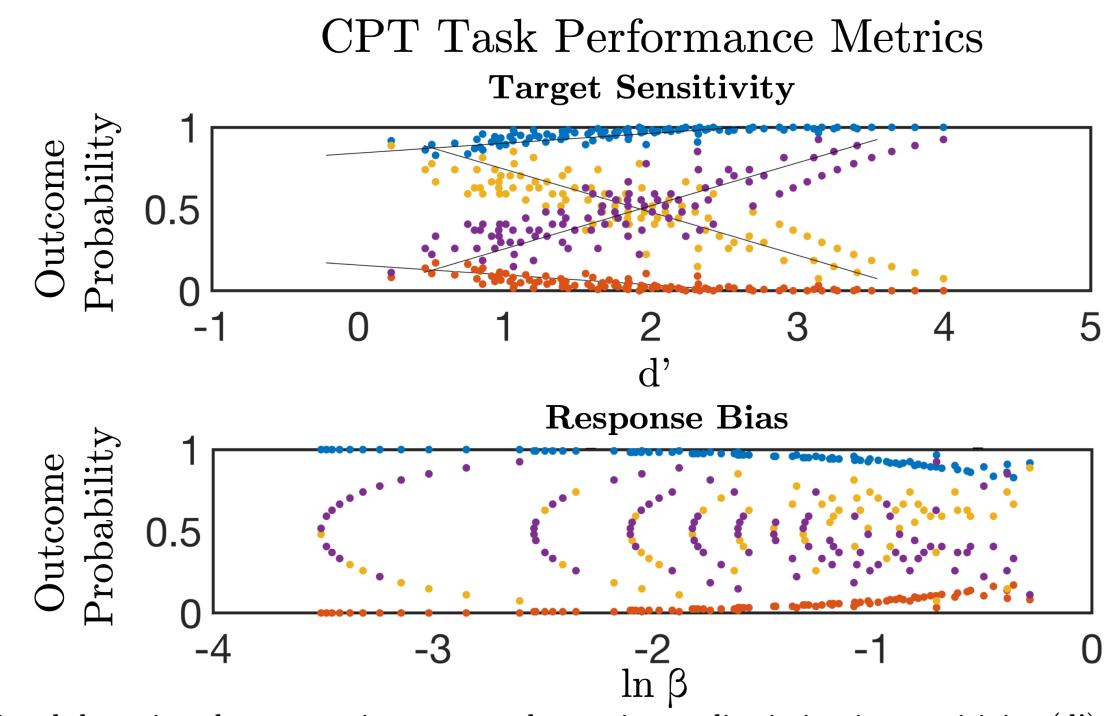

Figure 1 Signal detection theory metrics were used to estimate discriminative sensitivity (d') and overall response bias $(\ln \beta)$ for targets versus lures in the Continuous Performance Task (CPT). d' increased linearly with higher probability of a Correct Rejection (purple) and response to target (Hits, blue); and declined with greater False Alarm responses (yellow) and Miss rate (red) to targets (top). The probability of a Correct Rejection and response to target was a non-linear decreasing function of increasing response bias (bottom). Greater false alarm rates were indicative of elevated response bias and lower target-lure discrimination.

Figure 1:

Figure 2 Structural equation model of latent factors underlying inhibitory control (ICLF), risky reward processing (RRLF), and responsivity to positively (EPLF) and negatively salient emotional faces $(\mathrm{ENLF} ; \mathrm{RMSEA}=0.047$, TLI $=$ $0.926, \mathrm{CFI}=0.945)$. Inhibitory control was observed to be a significant effector of lower risk taking $(\mathrm{p}=0.026)$ and responsivity to both negative $(\mathrm{p}=0.007)$ and positive $(\mathrm{p}=0.02)$ emotional faces. No significant relationship was observed between RRLF and EPLF/ENLF. The ENLF manifested as fast and accurate responses to negative emotions, whereas EPLF was an indicator of longer looking times leading to correct recognition. ICLF effected faster looking time to all emotions at the expense of recognizing happy face expressions. Paths are faded to indicate statistical significance and strength of association. Numerical edge labels provide standardized estimates.

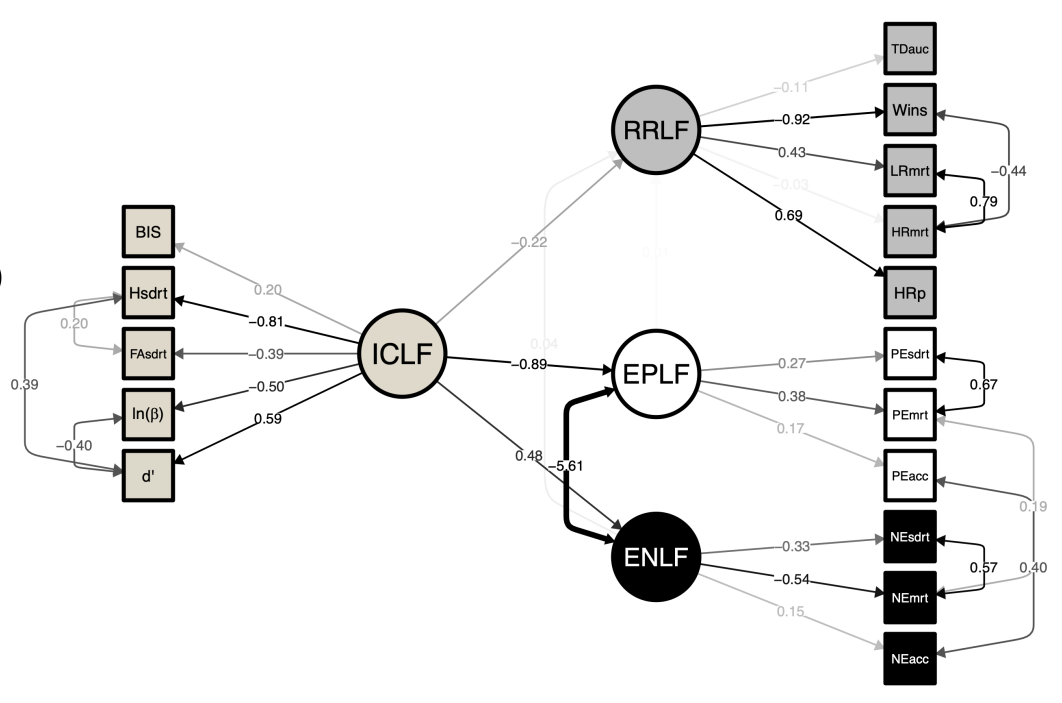

Figure 2: 


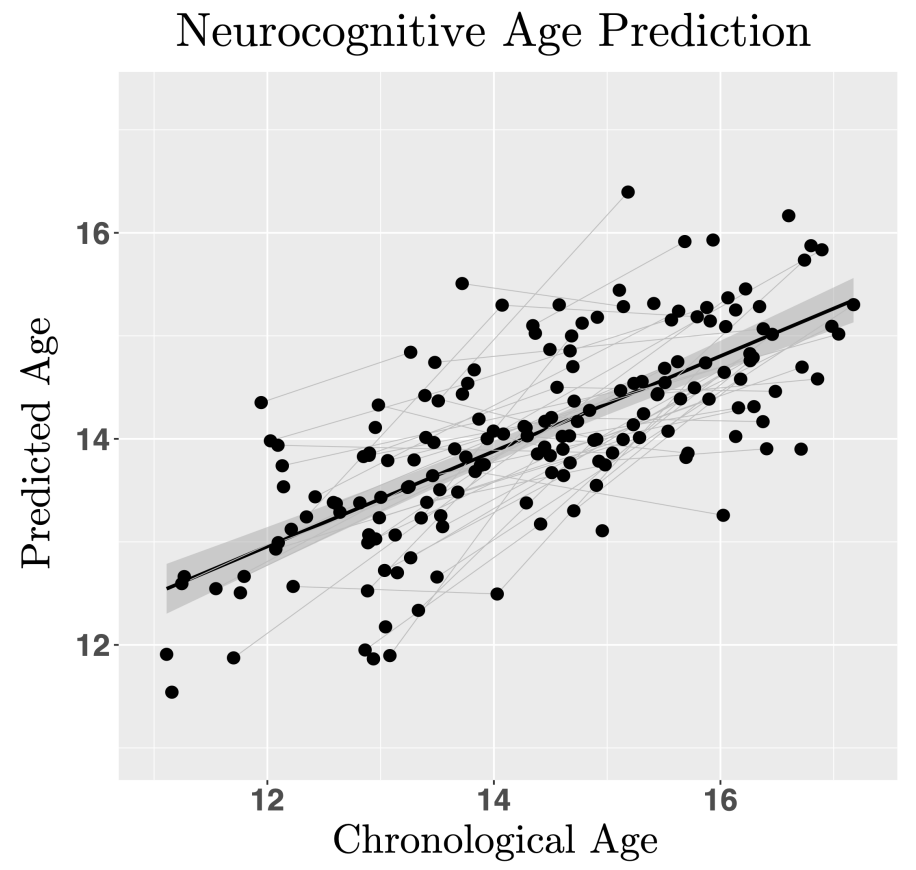

Figure 3 Regularized regression was used to estimate neurocognitive age in a training sample with leave-one-out cross validation to estimate linear model hyperparameters (lambda $=0.083 ; \mathrm{L} 2-$ norm ridge regression with alpha $=0$ ) minimizing mean squared error for predicting age with inhibitory control, risk/reward processing and emotional processing latent factors in a test sample (50\% participants split into train/test datasets). Model performance was assessed by computing the ratio between the mean cross-validated error and variance of observed age in the validation dataset $\left(\mathrm{R}^{2}=0.51\right)$. The neurocognitive maturity index is computed by subtracting the predicted neurocognitive age from the chronological age.

Figure 3:

Figure 4 Path Analysis was used to test the hypothesis that delayed cognitive maturation (CMI) effects greater pursuit of rewards/desires (BAS-D), which, in-turn, mediates the risk for violent outcomes in adulthood (DUSI-VP) determined by CMI. Pubertal development (PDS) and sex were significant covariates of cognitive maturation. Males with higher scale of physical development were more likely to show delayed cognitive maturation (i.e., lower inhibitory control, greater risk taking, emotion desensitization) compared to their peers. No significant effect was found between sex and risk. A negative correlation between Sex and PDS suggests females developed earlier or more quickly. Higher SES was found to ward off violence risk in adolescence. The structural model fit and full mediation $(\mathrm{Z}=-3.50, \mathrm{p}<$ 0.001) was found to be significant compared to a null model (RMSEA $=0.001, \mathrm{TLI}=1.070$, $\mathrm{CFI}=1.00)$. Dashed lines indicate estimation of free covariant parameters determined by

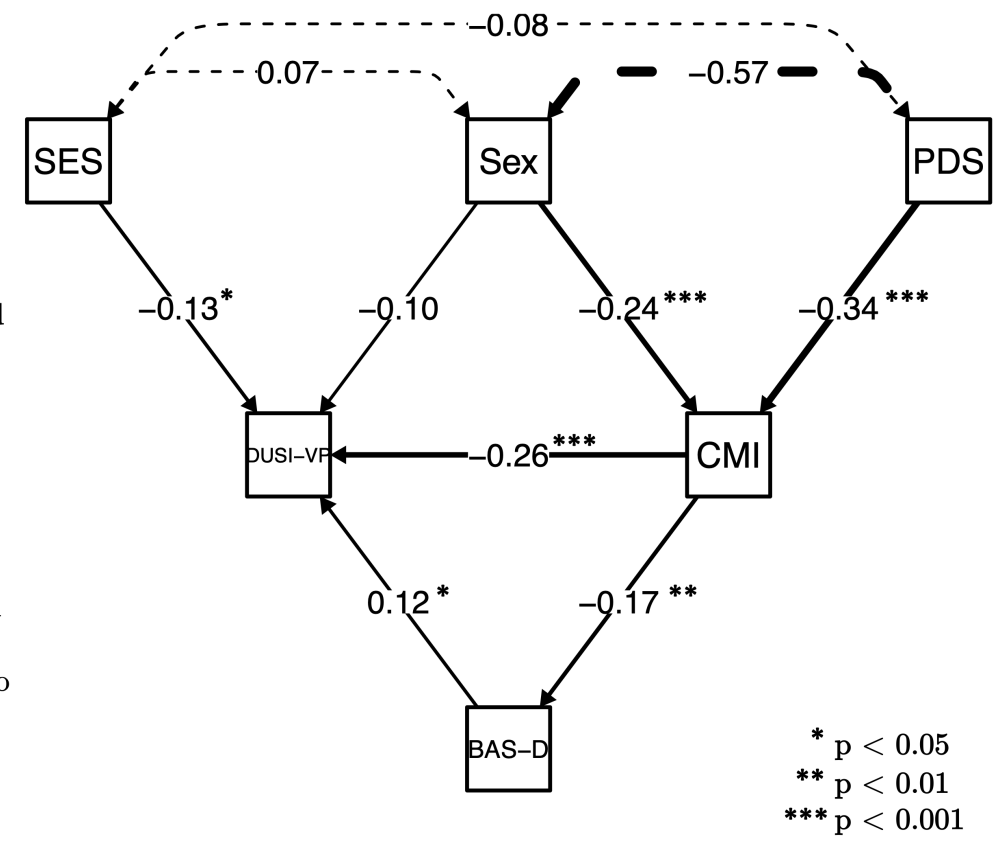
model fit. Asterisks indicate significance.

Figure 4: 